\title{
FAST DYNAMIC MESHING METHOD BASED ON DELAUNAY GRAPH AND INVERSE DISTANCE WEIGHTING INTERPOLATION
}

\author{
YIBIN WANG \\ College of Aerospace Engineering , Nanjing University of Aeronautics \\ and Astronautics, 29 Yudao road \\ Nanjing 210016, China \\ Yibin.wang@nuaa.edu.cn \\ NING QIN \\ Department of Mechanical Engineering, The University of Sheffield, \\ Sheffield S1 3JD, UK \\ n.qin@sheffield.ac.uk \\ NING ZHAO \\ College of Aerospace Engineering , Nanjing University of Aeronautics \\ and Astronautics, 29 Yudao road \\ Nanjing 210016, China
}

Published 22 June 2016

\begin{abstract}
A novel mesh deformation technique is developed based on the Delaunay graph mapping method and the inverse distance weighting (IDW) interpolation. The algorithm maintains the advantages of the efficiency of Delaunay-graph-mapping mesh deformation while possess the ability for better controlling the near surface mesh quality. The Delaunay graph is used to divide the mesh domain into a number of sub-domains. On each of the sub-domains, the inverse distance weighting interpolation is applied to build a much smaller sized translation matrix between the original mesh and the deformed mesh, resulting a similar efficiency for the mesh deformation as compared to the fast Delaunay graph mapping method. The paper will show how the near-wall mesh quality is controlled and improved by the new method while the computational time is compared with the original Delaunay graph mapping method.
\end{abstract}

Keywords: Dynamic mesh; inverse distance weighting; delaunay graph mapping.

\section{Introduction}

The dynamic mesh methods are widely used in computational fluid dynamics (CFD) simulations regarding shape deformation and moving. Two of the key issues of the method are the efficiency and mesh quality of the resultant mesh. During the shape

This is an Open Access article published by World Scientific Publishing Company. It is distributed under the terms of the Creative Commons Attribution 3.0 (CC-BY) License. Further distribution of this work is permitted, provided the original work is properly cited. 
deformation process, the mesh needs to be renewed repeatedly and therefore a fast dynamic mesh technique can efficiently decrease the computational time. For CFD simulation, the accuracy the result largely relies on the mesh quality, especially the mesh quality in the boundary layer, since in this region the solution derivative is relatively large and poor mesh can result in large truncation error. As a result, high quality and high efficiency are the most important aspects of the dynamic mesh techniques.

Dynamic mesh techniques can be generally classified into two main categories, based on either physical analogy or interpolation ${ }^{1}$. The physical analogy approach, normally requiring the connectivity information of the mesh, uses certain physics processes to propagate the mesh deformation from the boundary to the field mesh, such as the spring analogy approach first presented by Batina ${ }^{2}$. The spring analogy method treats each edge of the mesh as a spring which links adjacent nodes, hence the whole mesh can be viewed as a spring system, in which the stiffness is proportional to the reciprocal of the length. This method has been successfully applied to many unsteady and optimization problems but it is a relatively expensive approach due to the necessary iterations for solving the associated spring systems with high computational cost. It may also lead to invalid mesh for large deformation, in particular for viscous flow meshes. Later on, Farhat et al. ${ }^{3}$ introduced the torsional springs to prevent the mesh from becoming invalid. One of the genres requires to solve a set of partial differential equations, such as elastic analogies developed by Loehner and Yang ${ }^{45}$. Particularly, by solving a bi-harmonic set of equations, Helenbrook ${ }^{6}$ found that both mesh quality and orthogonality are superior to the Laplace equation based method. Generally, most of the physical analogy methods require relatively high computational cost; hence it is not efficient for mesh deforming problems requiring frequent deformation involving large meshes. Furthermore, large increment of deformation may result in poor or invalid meshes.

An interpolation method, by applying certain interpolation scheme, directly obtains the displacement or the new coordinates of each node. Two of the typical methods of this genre, are the Delaunay graph mapping method developed by Liu et al. ${ }^{7}$ and the radial basis function (RBF) method proposed by de Boer ${ }^{8}$. The Delaunay graph mapping method can be viewed as a fast interpolation scheme. However this method cannot well preserved the mesh quality near the wall. On the other hand, the RBF method, such as those used in fluid-structure interaction ${ }^{9,10}$ can generally better preserve the mesh quality near the boundary but the computational cost is much higher as the mesh size increases. Therefor several attempts was made to improve the efficiency of the RBF method. Rendall and Allen ${ }^{11}$ proposed an approximate RBF method with the data reduction algorithms. This method applies RBF interpolation based on a coarsened subset of the surface mesh. To decrease the error, a greedy algorithm was applied to add points from the surface mesh with the largest error. the performance of the RBF method is therefore improved significantly. However, this method may introduce surface error, hence they add a surface correction step to fix the problem ${ }^{12}$. Recently, Wang et al. ${ }^{13}$ developed a new method, which based on both Delaunay graph and RBF method. This method maintains the high efficiency of the Delaunay graph mapping method, while significantly 
improves the mesh quality for large deformation, especially the mesh near the wall is well preserved. Melville ${ }^{1415}$ developed an interpolation method based on connectivity to the moving surface, and applied it to aeroelastic simulations. By using the inversed distance as weight, Allen ${ }^{16}$ developed a similar technique, which the orthognality can be preserved by accounting for surface rotation. Witteveen and Bij $1^{17}$ proposed a direct interpolation strategy by using inverse distance weighted (IDW) interpolation, which is faster than the original RBF method. It requires to sum over all surface nodes for each mesh point, still resulting in high computational cost. Recently, Luke et al. ${ }^{1}$ proposed a fast explicit interpolation method, which has similar mesh quality as the RBF method, but with a relative faster speed. However this approach requires the user, to tune the input parameters according to their geometries. A novel method of dynamic mesh based on the Delaunay graph and inverse distance weighting (IDW) is presented in this paper. This method maintains the effectiveness of the original Delaunay graph mapping method, while possessing the ability of controlling the quality of the meshes near the boundary.

\section{Delaunay graph based IDW method}

\subsection{Delaunay graph mapping method}

The Delaunay graph mapping method ${ }^{7}$ as an efficient mesh deformation method has been widely used for fluid structure interaction and aeroelastic problems, flapping wing simulations and aerodynamic optimization. For relatively small deformation problems, this method can be successfully applied with high efficiency with good mesh quality. For example, Van der Burg et al. ${ }^{18}$ applied it to the aeroelastic problem in a high lift study, in which the mesh deformation could be done "in a couple of minutes" for a large 3D problem with 7 million tetrahedral cells using the Delaunay graph mapping method. Zhang et al. ${ }^{19}$ and Lee et al. ${ }^{20}$ used it effectively for bio-fluid and flapping wing simulations for efficient mesh deformation. Wang et al. ${ }^{21}$ applied it for shock control aerodynamic shape optimization. However, for large deformation, especially for deformation with large rotation, the mesh quality can degrade or even becomes invalid. In addition, due to the lacking of the mesh quality control, the mesh quality near the wall quickly degrades as the mesh deformation.

\subsection{Inverse distance weighting Interpolation}

The IDW interpolation method is first developed by Witteveen and Bij $1^{17}$. It is an explicit method for multivariate interpolation of scattered points. The displacement of the node can be calculated by

$$
s(\mathrm{x})=\frac{\sum_{i=1}^{n_{d}} S_{i} r_{i}^{-c_{d}}+\sum_{i=1}^{n_{s}} S_{i} r_{i}^{-c_{s}}}{\sum_{i=1}^{n_{d}} r_{i}^{-c_{d}}+\sum_{i=1}^{n_{s}} r_{i}^{-c_{s}}}
$$

where $n_{d}$ is the total number of dynamic boundary nodes, $n_{z}$ is the total number of static boundary nodes, $\mathrm{r}$ is the Euclidian distance. $c_{d}$ and $\varepsilon_{z}$ are shape parameters. For different 
motion the $r_{d}$ and $r_{s}$ are different. The resultant mesh quality highly depends on the shape parameters, however, the proper setting of these parameters varies from cases to cases, therefore it is difficulty for beginner to properly choose the parameters. In addition, it requires to sum over all surface nodes for each mesh point, still resulting in high computational cost. The motivation of this paper is to developed a novel method to significantly improve the efficiency of the IDW method, and to avoid the user define parameters.

\subsection{The procedures of Delaunay graph based IDW method}

The procedure of the Delaunay graph based IDW method (DG-IDW) is similar to the original Delaunay graph mapping method (DGM). The main difference is in the last step, the IDW function is used to map the nodes rather than that based on surface or volume ratios. The algorithm is set out in the following procedure:

(a) Generating the Delaunay graph by using all the boundary nodes of the original mesh;

(b) Locating the mesh points in the graph;

(c) Moving the Delaunay graph according to the specified geometric motion/deformation;

(d) Mapping the mesh points in the new graph according to the IDW interpolation. The displacement of each node in the Delaunay triangle is

$$
s(x)= \begin{cases}\frac{a \sum_{i=1}^{2} s_{i} r_{i}^{-2}}{a \sum_{i=1}^{2} r_{i}^{-2}+2 r^{-1}} & n s=1 \\ \frac{2 a s_{s} r^{-2}}{2 a r^{-2}+\sum_{i=1}^{n_{s}} r_{i}^{-1}} & n s=2 \\ \frac{a \sum_{i=1}^{n_{d}} s_{i} r_{i}^{-2}+\sum_{i=1}^{n_{s}} s_{i} r_{i}^{-1}}{a \sum_{i=1}^{n_{d}} r_{i}^{-2}+\sum_{i=1}^{n_{s}} r_{i}^{-1}} & \text { else }\end{cases}
$$

where $\mathrm{a}=7$ for translation, $\mathrm{a}=1.4$ for rotation.

Steps 1-3 are exactly the same as the original Delaunay graph mapping method; hence the details of these steps are skipped in this paper. More details can be found in Ref 7. In Step 4, the IDW interpolation is used to calculate the displacement of the internal mesh nodes from the given displacement of the Delaunay triangle nodes on the boundary, while the original Delaunay mapping method uses surface or volume ratios to calculate the displacement of inner nodes.

\section{Results and discussions}

\subsection{Test case 1: rotation and translation}

The first test case is a structured mesh which contains 2340 nodes, shown in Fig. 1. The inner rectangle was rotated around its center by $30^{\circ}, 45^{\circ}, 60^{\circ}, 90^{\circ}$, respectively. For all the test cases in this paper, the meshes were generated by only one mapping for fair 
comparison. The mesh quality were compared with the Delaunay graph mapping method (DGM), inversed distance weighting method and radial basis function method in Tables 1 and 2. As can been seen from the tables, the IDW method gives the best mesh quality for both averaged and minimal mesh quality; while the Delaunay graph based inversed distance weighting method (DGIDW) shows second best averaged and minimal mesh quality. In Table 3, the CPU time of the four methods are compared. The DGM and DGIDW shows best efficiency, the CPU time of the two methods is less than $0.001 \mathrm{~s}$, while the CPU time of the IDW and RBF methods is one order larger than the DGM and DGIDW method. In Fig. 3, the resultant mesh by four methods are shown, which rectangle in the middle was rotated by $45^{\circ}$. As shown in the figures, only the DGM method fails to keep the mesh quality near the wall, while rest of the methods all maintain the mesh quality in this region.

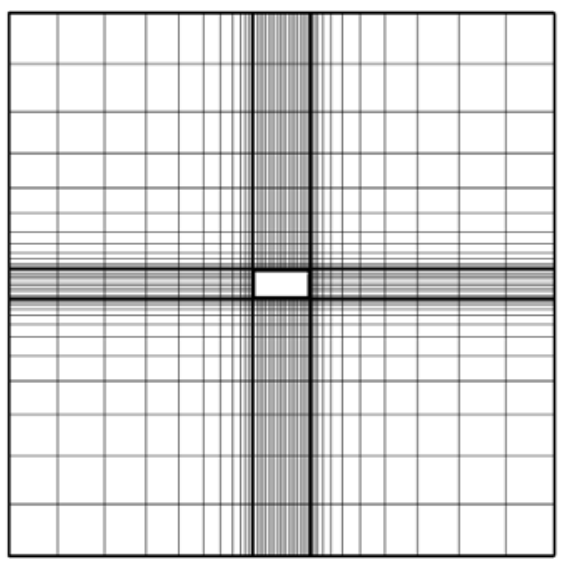

Fig. 1. Original mesh.

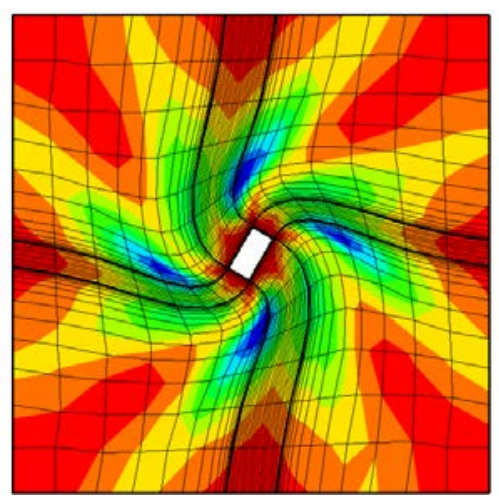

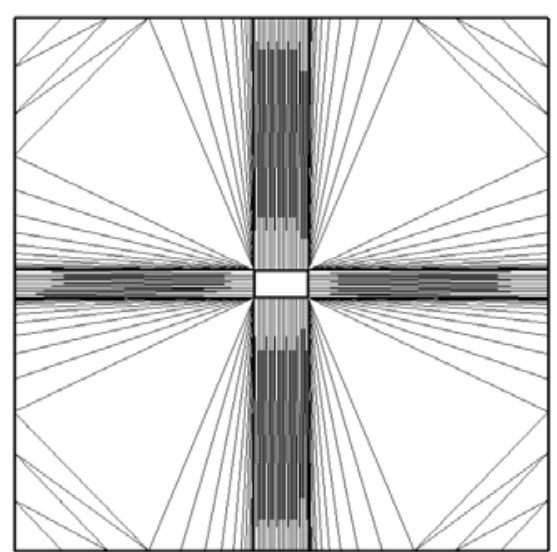

Fig. 2. Original Delaunay graph.

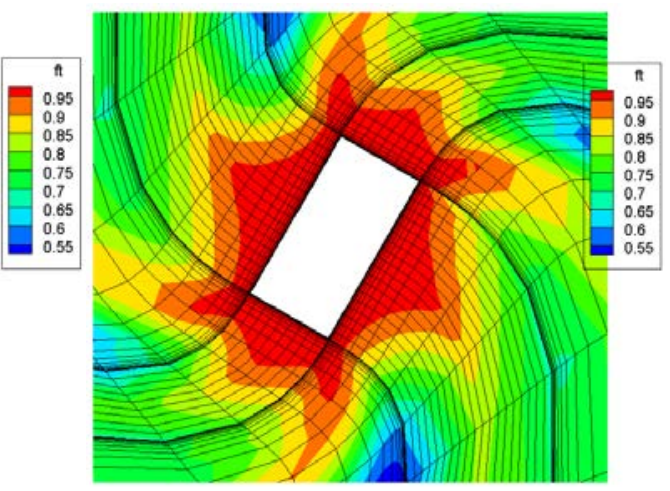

(a) DGIDW 

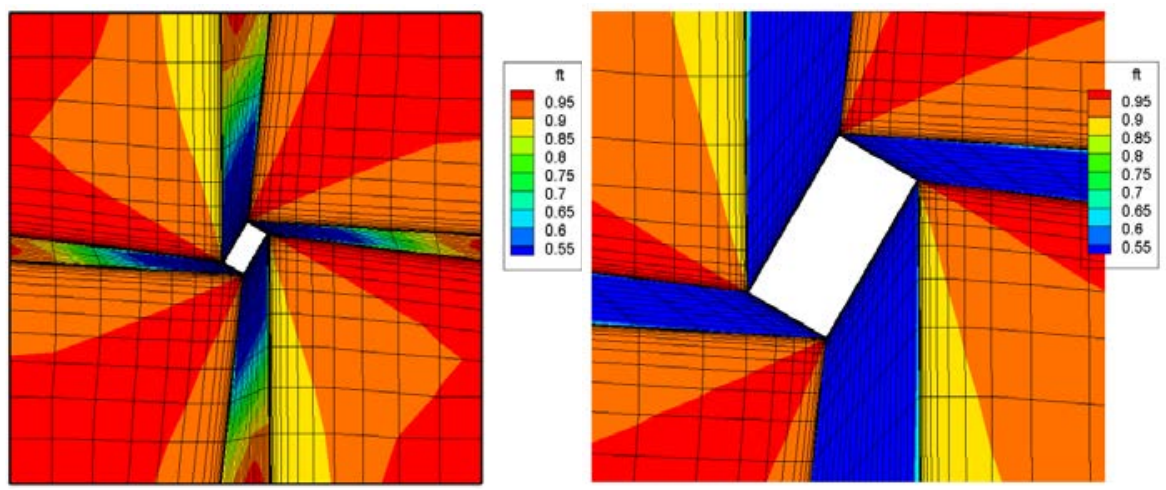

(b) DGM
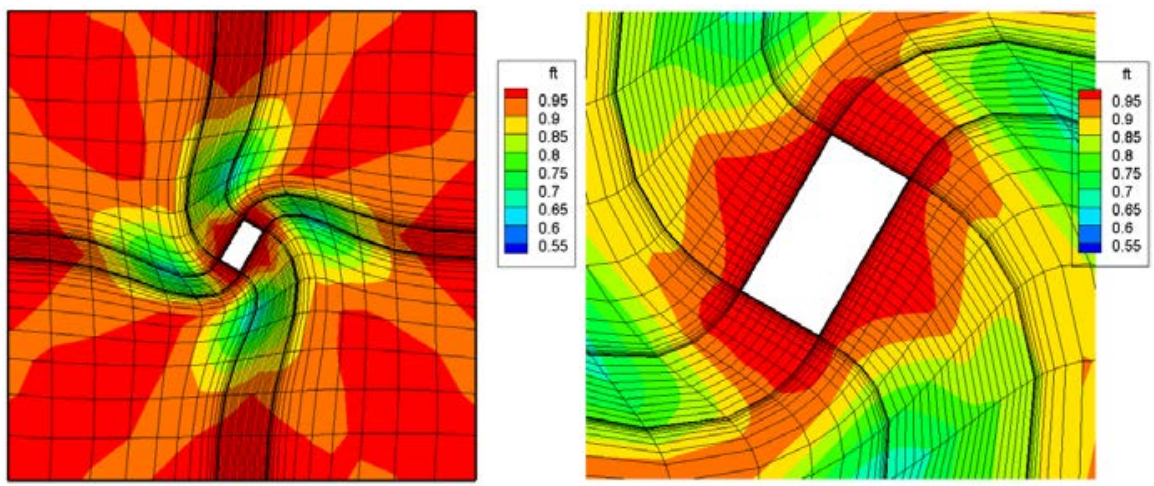

(c) IDW
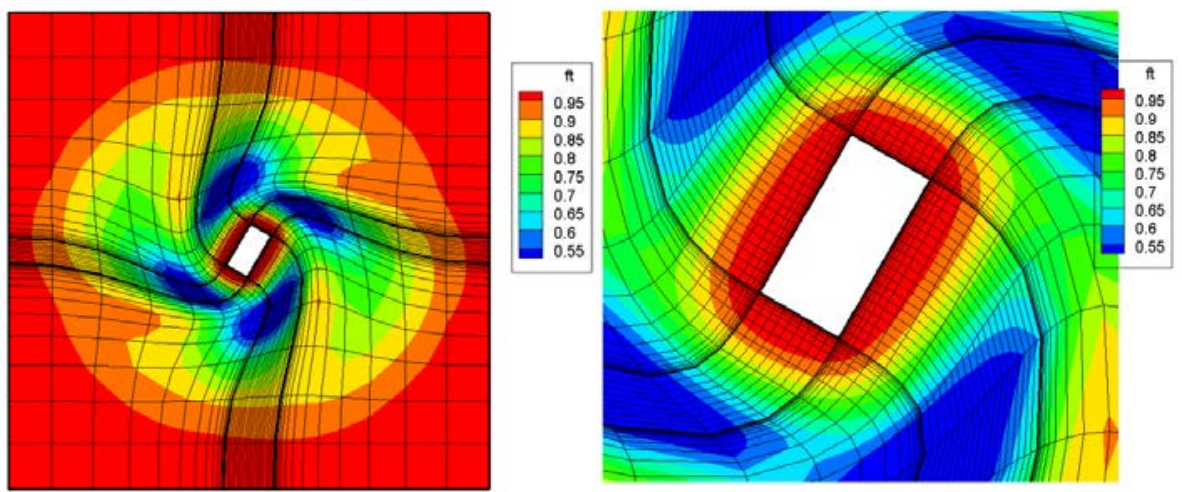

(d) RBF

Fig. 3. The deformed mesh by different methods (rotation by $45^{\circ}$ ). 
Table 1 Averaged mesh quality.

\begin{tabular}{lllll}
\hline & DGM & IDW & RBF & DG-IDW \\
\hline $\mathbf{3 0}^{\circ}$ & 0.885 & 0.970 & 0.954 & 0.952 \\
$\mathbf{4 5}^{\circ}$ & 0.781 & 0.946 & 0.907 & 0.920 \\
$\mathbf{6 0}^{\circ}$ & 0.676 & 0.919 & 0.851 & 0.884 \\
$\mathbf{9 0}^{\circ}$ & 0.341 & 0.863 & 0.739 & 0.814 \\
\hline
\end{tabular}

Table 2 Minimal mesh quality.

\begin{tabular}{lllll}
\hline & DGM & IDW & RBF & DG-IDW \\
\hline $\mathbf{3 0}^{\circ}$ & 0.684 & 0.882 & 0.784 & 0.784 \\
$\mathbf{4 5}^{\circ}$ & 0.408 & 0.768 & 0.580 & 0.627 \\
$\mathbf{6 0}^{\circ}$ & 0.159 & 0.641 & 0.363 & 0.469 \\
$\mathbf{9 0}^{\circ}$ & -0.571 & 0.396 & 0.356 & 0.208 \\
\hline
\end{tabular}

Table 3 CPU time.

\begin{tabular}{lllll}
\hline & DGM & IDW & RBF & DG-IDW \\
\hline $\mathbf{3 0}^{\circ}$ & $<0.001$ & 0.015 & 0.065 & $<0.001$ \\
$\mathbf{4 5}^{\circ}$ & $<0.001$ & 0.016 & 0.062 & $<0.001$ \\
$\mathbf{6 0}^{\circ}$ & $<0.001$ & 0.016 & 0.062 & $<0.001$ \\
$\mathbf{9 0}^{\circ}$ & $<0.001$ & 0.016 & 0.063 & $<0.001$ \\
\hline
\end{tabular}

For the same configuration and initial mesh, the inner rectangle was moved 2 units both up and to the right. The mesh quality were compared in Table 4 . For the translation, the DGM method shows best minimal mesh quality, while its averaged mesh quality is second worst; the RBF method gives high averaged mesh quality but with worst minimal mesh quality; both average and minimal mesh quality for IDW method is ranked in the third place. The DGIDW method shows best averaged mesh quality and second best minimal mesh quality, therefore the DGIDW method is more suitable for the translation motion. In Fig. 4, the four resultant mesh were shown with the mesh quality contours. Both the RBF and the DGIDW methods kept the mesh quality near the moving boundary, while the DGM and IDW methods failed to maintain the mesh quality in the same region. The CPU time were also compared in the Table 4, the DGIDW and the DGM are the most efficient method among the four. 

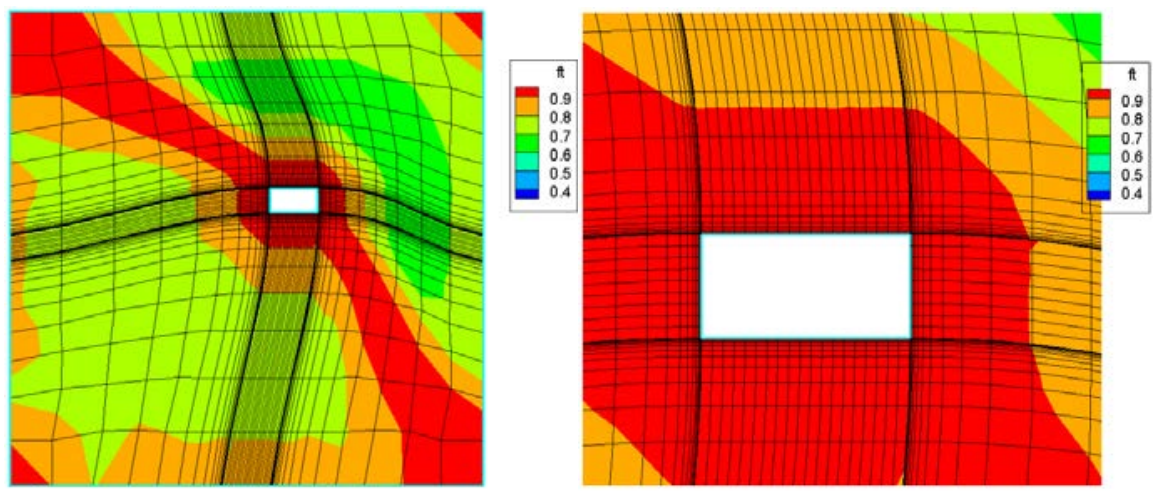

(a) DGIDW
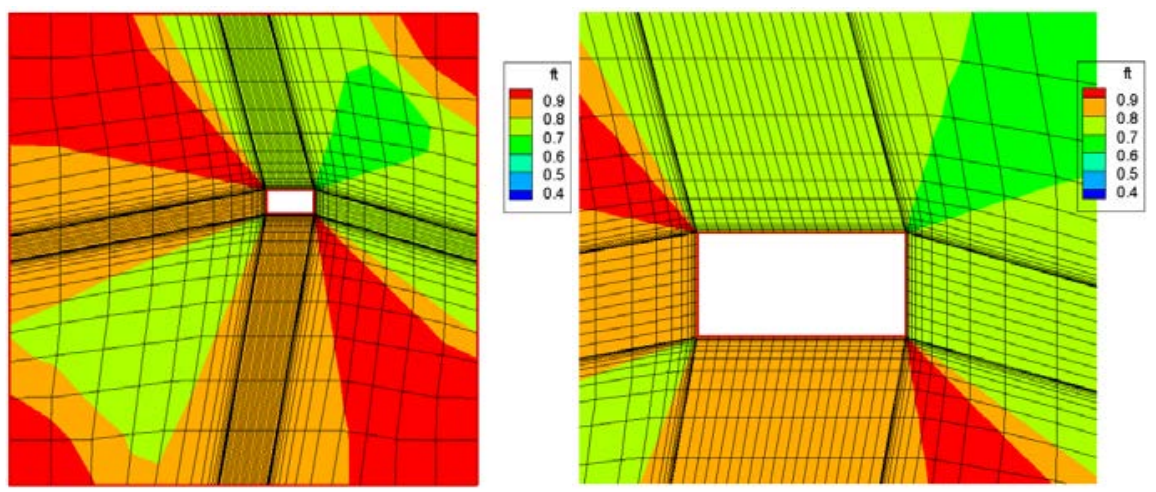

(b) DGM
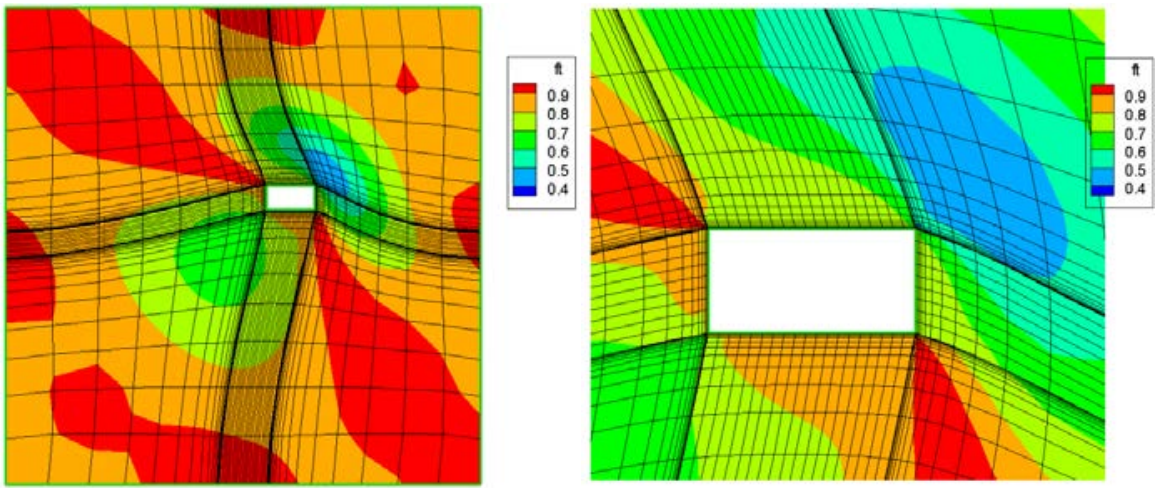

(c) IDW 

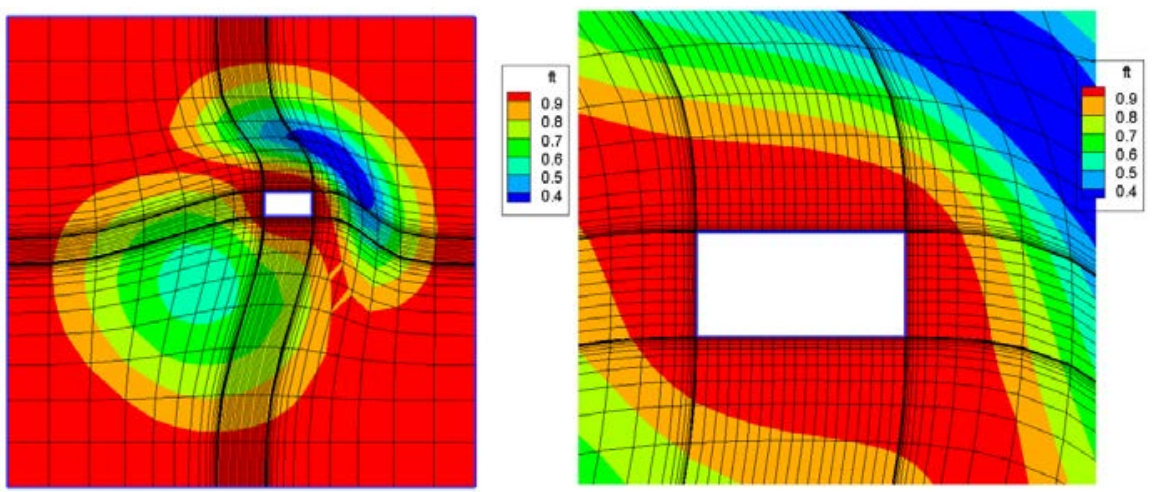

(d) $\mathrm{RBF}$

Fig. 4. The resultant mesh by different method (translation).

Table 4 mesh quality and CPU time comparison.

\begin{tabular}{lllll}
\hline & DGM & IDW & RBF & DG-IDW \\
\hline averaged & 0.792 & 0.777 & 0.860 & 0.881 \\
minimal & 0.649 & 0.422 & 0.223 & 0.591 \\
CPU time & 0.001 & 0.033 & 0.107 & 0.001 \\
\hline
\end{tabular}

\subsection{Test case 2: unstructured mesh for NACA0012 aerofoil}

An unstructured mesh with 1647 nodes and 3054 cells for NACA0012 aerofoil was used as the second test case. The original mesh and its Delaunay graph were shown in Figs. 5 and 6 . In order to test the capability of the four methods, a sliver cell was put right behind the trailing edge, as shown in Fig. 6(c). Any slight degradation of the mesh quality may cause the cell become invalid. The aerofoil was moved 2 units both up and to the right. The deformed mesh are shown in Figs. 7 and 8. As can been seen form the figures, the RBF, DGIDW and IDW method maintains the mesh quality near the aerofoil, while the DGM method largely changed the mesh quality in the same region. Particularly, in Fig. 8 the mesh near the trailing edge is shown. the mesh generated by the DGM method become invalid due to the overlapping. In Table 5, the mesh quality were compared, the DGIDW method shows best mesh quality for both averaged and minimal mesh quality. 

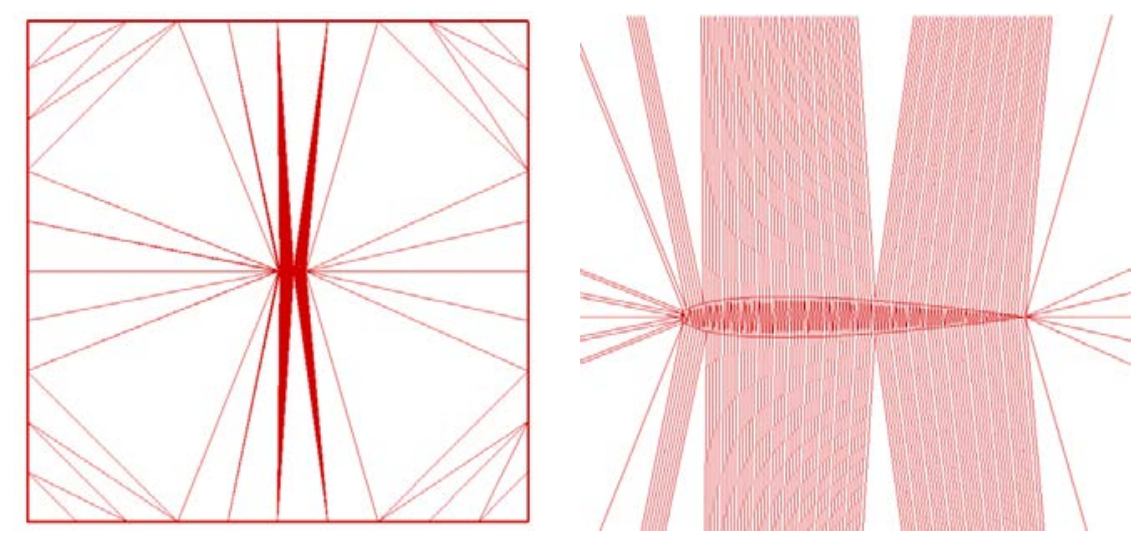

Fig. 5. Original Delaunay Graph.

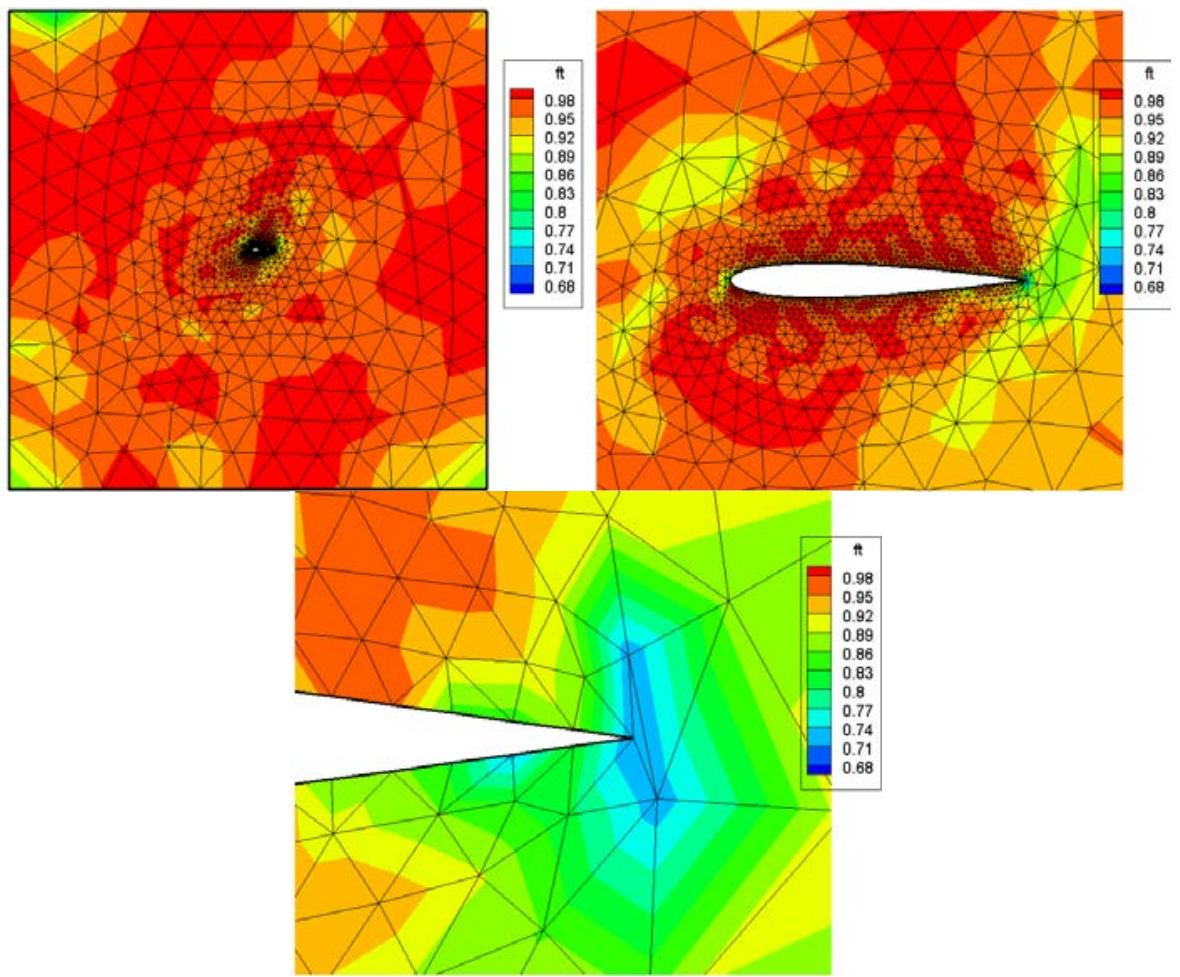

Fig. 6. Original mesh.

Table 5 Mesh quality comparison.

\begin{tabular}{llllll}
\hline & DGM & IDW & RBF & DG-IDW & original \\
\hline averaged & 0.736 & 0.895 & 0.895 & 0.908 & 0.965 \\
minimal & -0.185 & 0.116 & 0.176 & 0.176 & 0.177 \\
\hline
\end{tabular}


Fast Dynamic Meshing Method
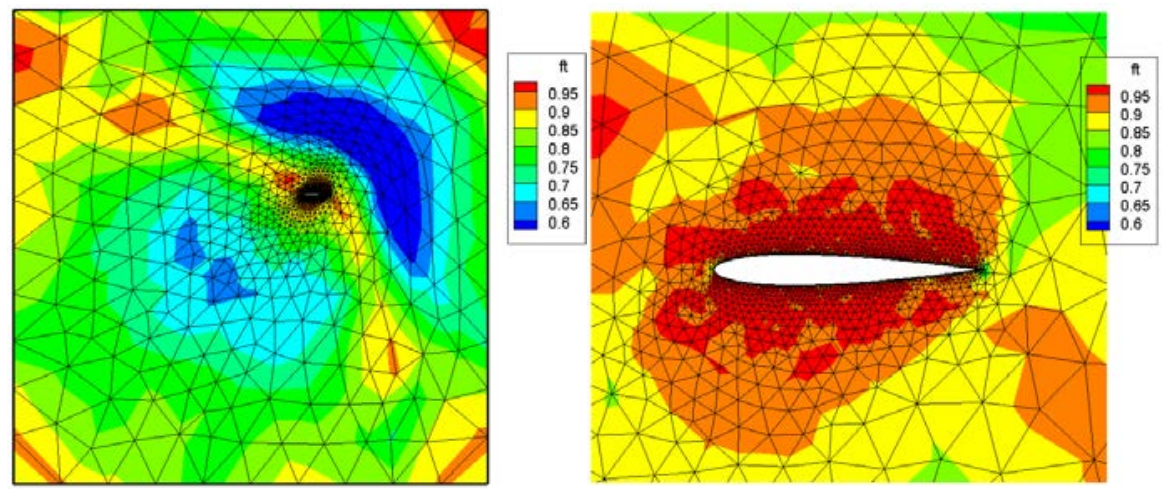

(a) DGIDW
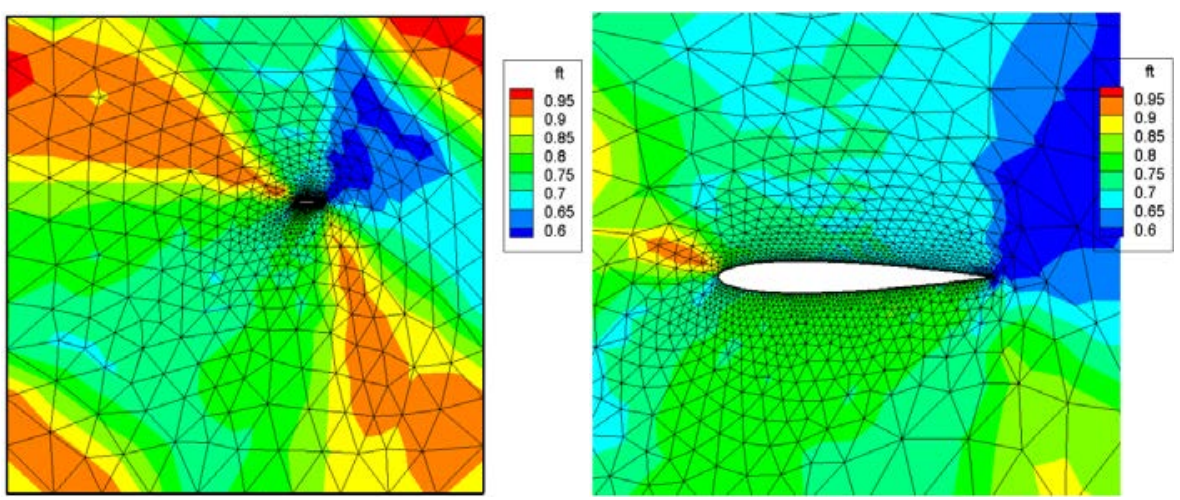

(b) DGM
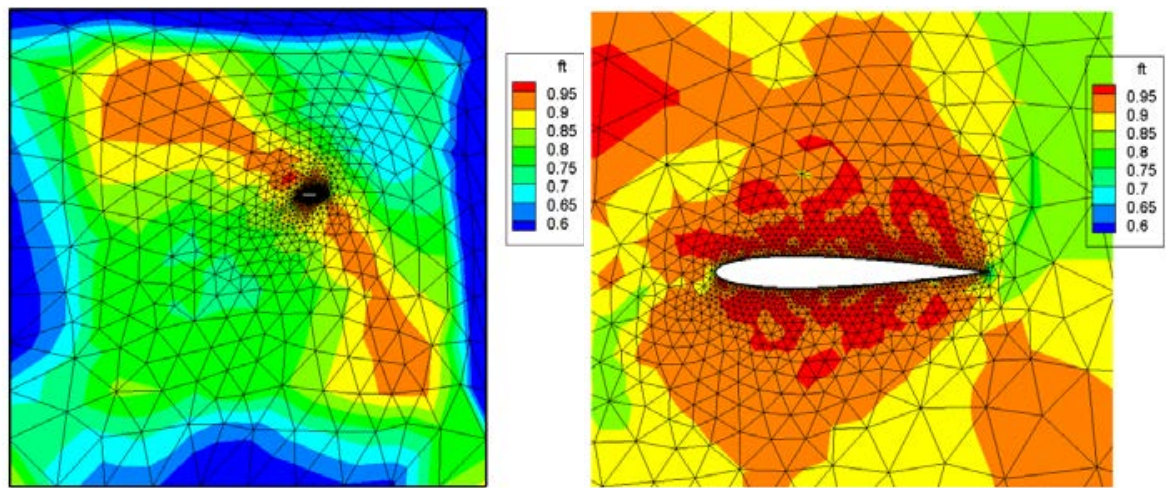

(c) IDW 

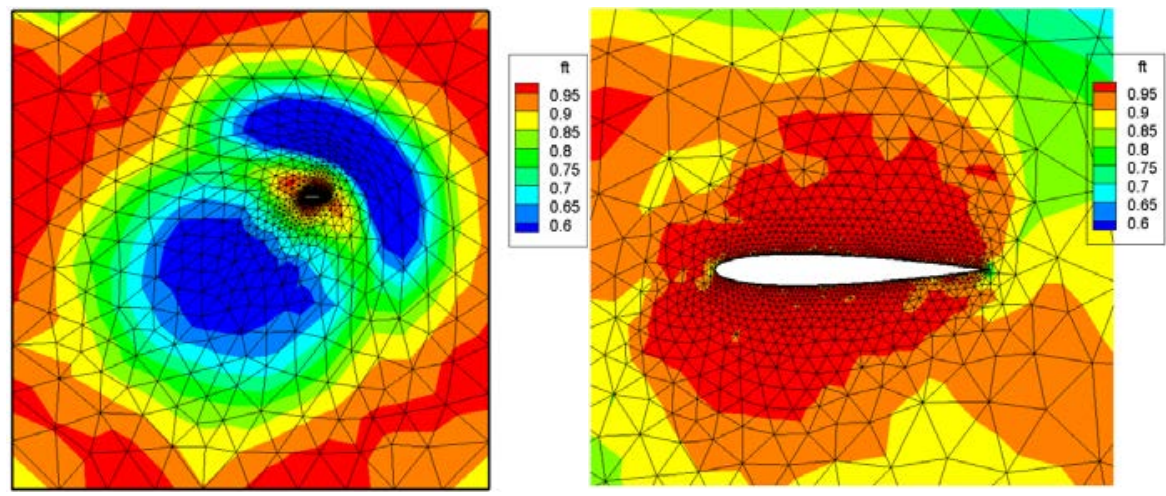

(d) $\mathrm{RBF}$

Fig. 7. The resutant mesh by different method (translation).

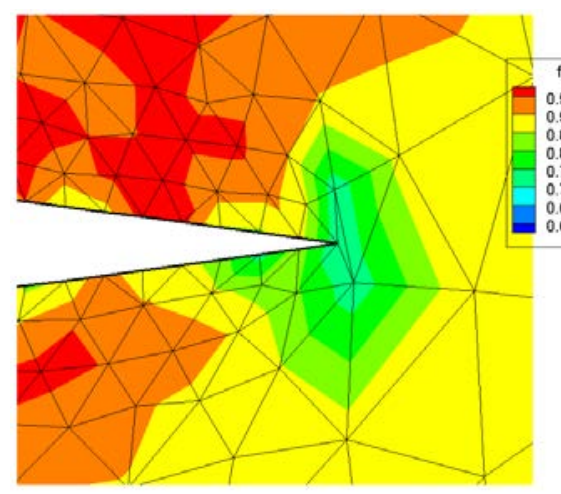

(a) DGIDW

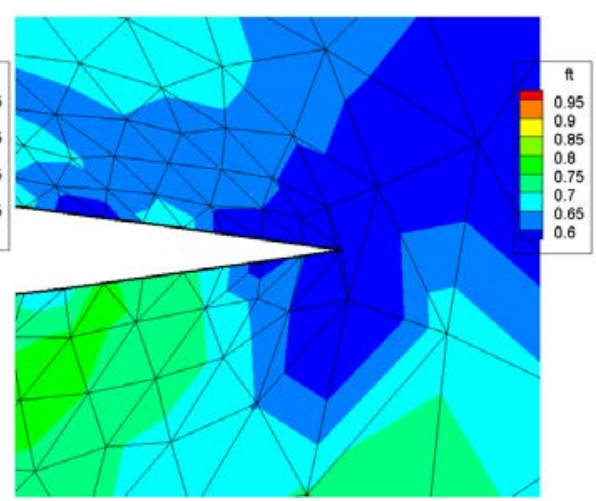

(b)DGM

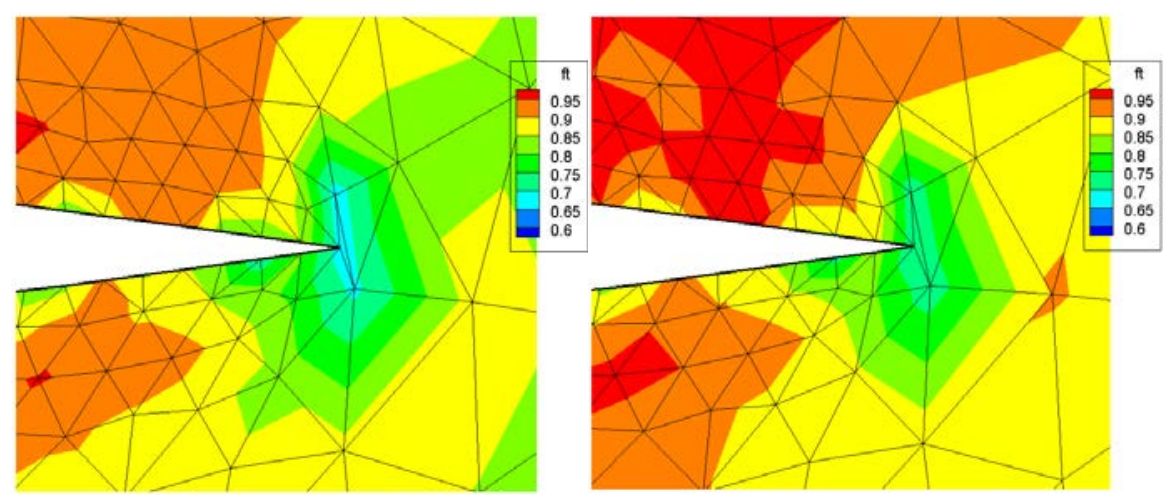

(c) idw

(d) RBF

Fig. 8. The mesh quality contour near the trailing edge (translation).

The aerofoil were then rotated by $45^{\circ}$. The deformed mesh were shown in Fig. 9 and 10. For the rotation, the DGM method fails to retain the mesh quality near the aerofoil. the low quality cells are all around the aerofoil, which may cause significant numerical 
error in the CFD simulation; while the IDW, RBF and DGIDW methods retain the mesh quality near the aerofoil. for the rotation, the sliver cell behind the trailing edge did not become invalid for all the method after the mapping. In Table 6 , the mesh quality are compared, the new method i.e. DGIDW method shows the best results for both averaged and minimal mesh quality.
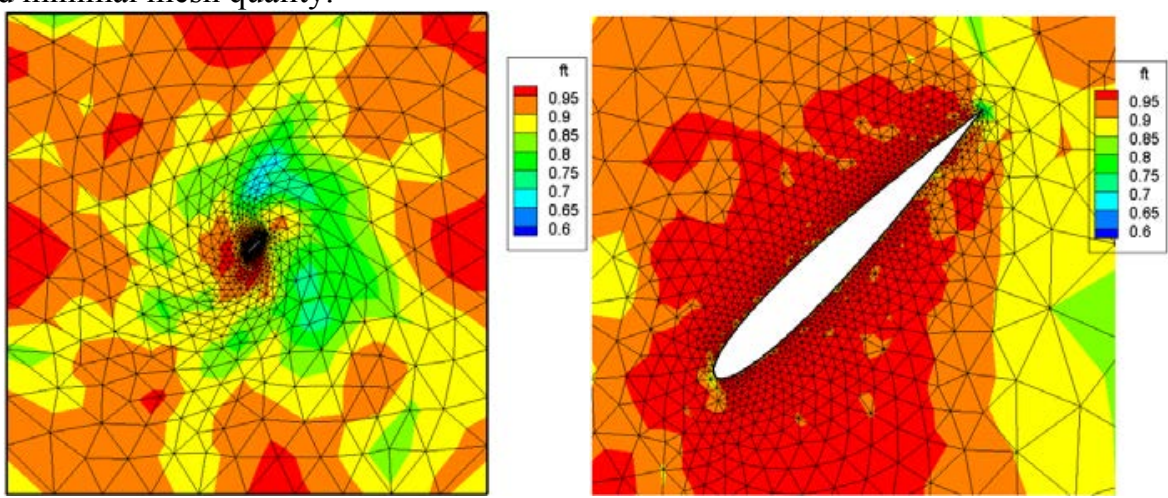

(a) DGIDW
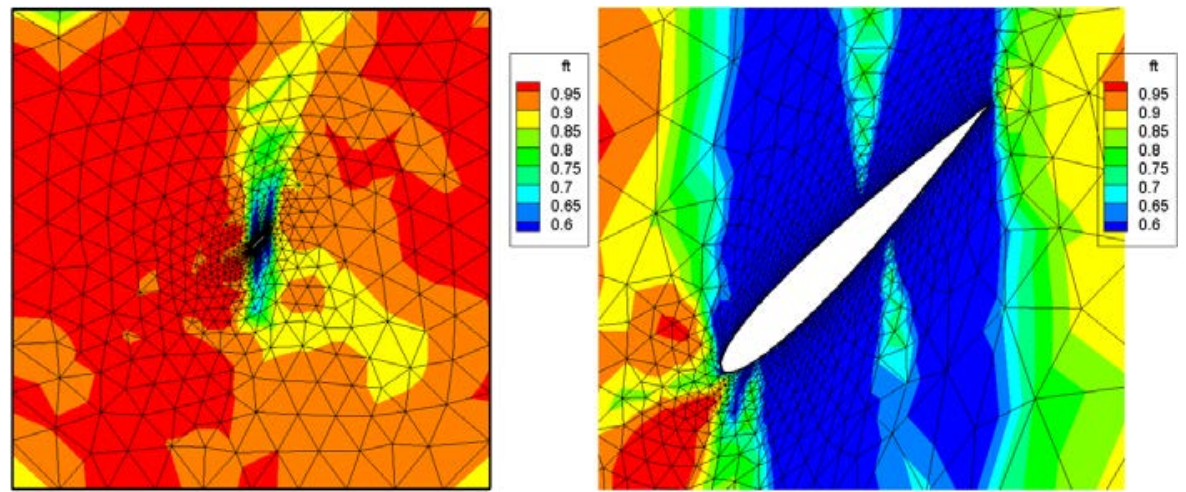

(b) DGM
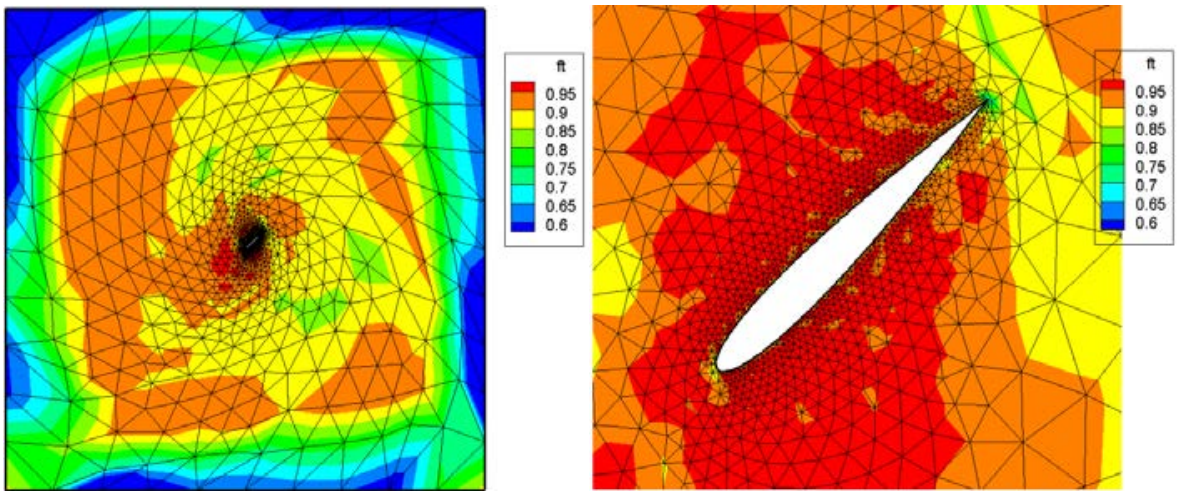

(c) IDW 

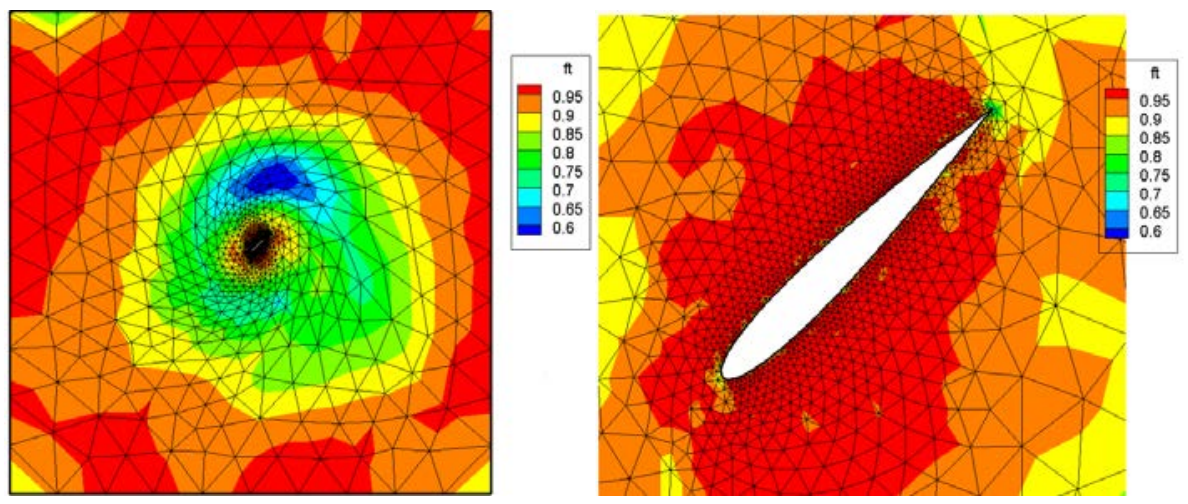

(d) $\mathrm{RBF}$

Fig. 9 the deformed mesh by different method (rotation)

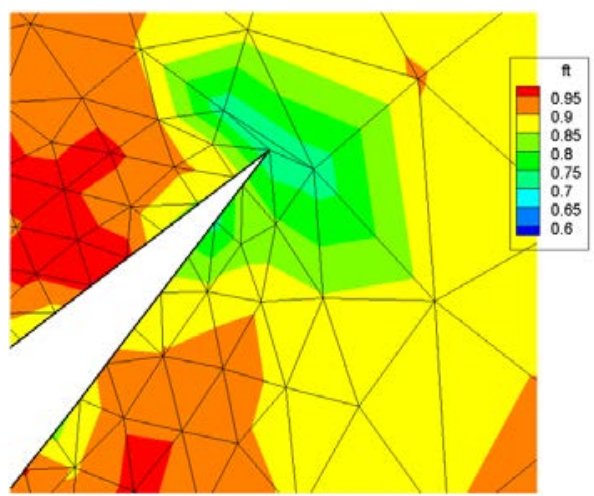

(a) DGIDW

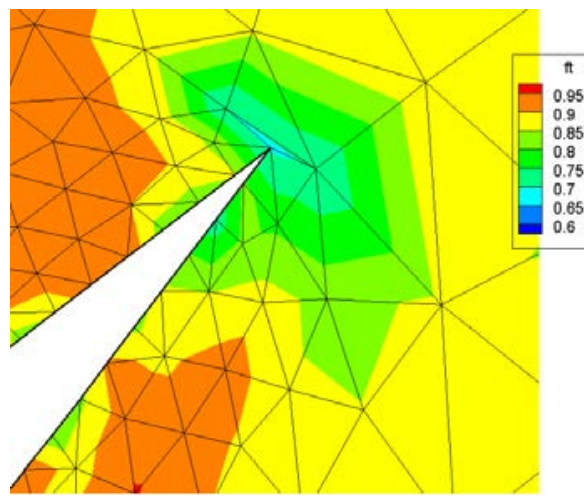

(c) IDW

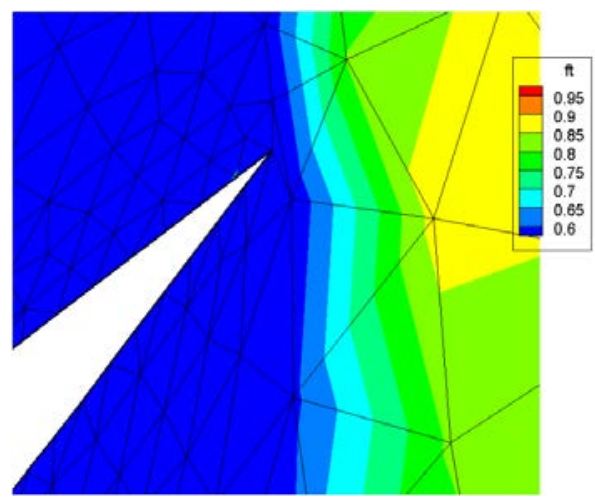

(b)DGM

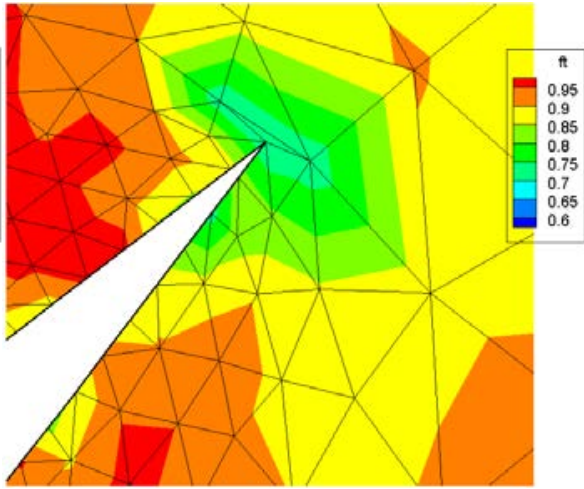

(d) RBF

Fig. 10. The mesh quality contour near the trailing edge (rotation). 
Table 6 Mesh quality comparison

\begin{tabular}{llllll}
\hline & DGM & IDW & RBF & DG-IDW & original \\
\hline averaged & 0.942 & 0.927 & 0.923 & 0.933 & 0.965 \\
minimal & 0.151 & 0.162 & 0.176 & 0.177 & 0.177 \\
\hline
\end{tabular}

\section{Conclusions}

A novel dynamic mesh deformation method is developed based on a combination of the Delaunay graph mapping method with inverse distance weighted interpolation. Since it is a point-by-point method, it can be applied for all types of grids, structured or unstructured. It maintains the high efficiency of the original Delaunay graph mapping method, while improves the mesh quality near the boundaries, which is very important for high Reynolds number CFD simulation. By separating interpolations for translation and rotation motion, the DG-IDW method can preserve the mesh quality near the boundary even when the rotation is extremely large, which is a significant improvement of the original Delaunay graph mapping method when very large rotation is present. The cost of the algorithm is the same order as the Delaunay graph mapping method.

\section{Acknowledgement}

This work is partially funded by the Priority Academic Program Development of Jiangsu Higher Education Institutions of China national nature science foundation of China 11432007 and nature science foundation of Jiangsu Province BK20140805.

\section{References}

1. E. Luke, E. Collins, E. Blades, A fast mesh deformation method using explicit interpolation, J. Comput. Phys. 231(2012) 586-601.

2. J.T. Batina, Unsteady Euler algorithm with unstructured dynamic mesh for complexaircraft aerodynamic analysis, AIAA J. 29 (3) (1991) 327-333.

3. C. Farhat, C. Degand, B. Koobus, M. Lesoinne, Torsional springs for two-dimensional dynamic unstructured fluid meshes, Comput. Method. Appl. M. 163 (1998) 231-245.

4. R. Loehner, C. Yang, Improved ALE mesh velocities for moving bodies, Commun.Numer. Meth. En. 12 (1996) 599-608.

5. J.D. Bau, H. Luo, R. Loehner, E. Goldberg, A. Feldhun, Application of unstructured moving body methodology to the simulation of fuel tank separation from an F-16 fighter, in: 35th Aerospace Sciences Meeting and Exhibit, AIAA Paper No. AIAA-1997-0166, Reno, NV, 1997.

6. B. Helenbrook, Mesh deformation using the biharmonic operator, Int. J. Numer. Meth. Eng. 56 (2003) 1007-1021.

7. X. Liu, N. Qin, H. Xia, Fast dynamic grid deformation based on delaunay graph mapping, J. Comput. Phys. 211 (2006) 405-423.

8. A. de Boer, M. van der Schoot, H. Bijl, Mesh deformation based on radial basis function interpolation, Comput.Struct. 85 (2007) 784-795. 
9. A. Beckert, H. Wendland, Multivariate interpolation for fluid-structure-interaction problems using radial basis functions, Aerospace Science and Technology 5 (May-June) (2001) 125-134.

10. R. Ahrem, A. Beckert, H. Wendland, A meshless spatial coupling scheme for large-scale fluid-structure interaction problems, Computer Modeling in Engineering and Sciences 12 (2006) 121-136.

11. T. Rendall, C. Allen, Efficient mesh motion using radial basis functions with data reduction algorithms, J. Comput. Phys. 228 (17) (2009) 6231-6249.

12. T. Rendall, C. Allen, Parallel efficient mesh motion using radial basis functions with application to multi-bladed rotors, Int. J. Numer. Meth. Eng. 81 (1) (2010) 89-105.

13. Y. Wnag, N, Qin, N. Zhao. Delaunay graph and radial basis function for fast quality mesh deformation. Journal of Computational Physics, Volume 294, 1 August 2015, 149-172

14. R. Melville, Dynamic aeroelastic simulation of complex configurations using overset grid systems, in: Fluids 2000, AIAA, Denver, AIAA Paper 2000-2341, 2000.

15. R. Melville, Nonlinear simulation of F-16 aeroelastic instability, in: 39th AIAA Aerospace Sciences Meeting, AIAA, Reno, NV, AIAA Paper 2001-0570, 2001.

16. C.B. Allen, Parallel universal approach to mesh motion and application to rotors in forward flight, Int. J. Numer. Meth. Eng. 69 (10) (2007) 2126 - 2149.

17. J.A.Witteveen, H. Bijl, Explicit mesh deformation using inverse distance weighting interpolation, in: 19th AIAA Computational Fluid Dynamics Conference, AIAA, San Antonio, Texas, AIAA Paper 2009-3996, 2009.

18. J.W. Van Der Burg, H. Freiherr Von Geyr, R. Heinrich, P. Eliasson, T. Delille, and J. Krier, Geometrical Installation and Deformation Effects in High-Lift Flows, AIAA Journal,47(2009), 60-70

19. L. Zhang,X. Chang,X. Duan,Z.He, Applications of dynamic hybrid grid method for threedimensional moving/deforming boundary problems. Comput. Fluids, 62 (2012), 45-63

20. K.B. Lee, J.H. Kim, C. Kim, Aerodynamic Effects of Structural Flexibility in TwoDimensional Insect Flapping Flight, J of Aircraft, 48(3), (2011),894-909.

21. H. Wang, J. Leskinen, D. Lee, Active flow control of airfoil using mesh/meshless methods coupled to hierarchical genetic algorithms for drag reduction design, Engineering Computations,30(4),(2013),562-580. 